\title{
Identification of longitudinal coherent oscillations induced by path-length fluctuations
}

\author{
Yoshihiko Shoji \\ Laboratory of Advanced Science and Technology for Industry (LASTI), University of Hyogo, 1-1-2 Kouto, Kamigori-cho, Ako-gun, \\ Hyogo 678-1205, Japan
}

(Received 8 May 2007; published 15 January 2008)

\begin{abstract}
This paper explains an analytical formula for longitudinal coherent oscillations in an electron storage ring, including path-length oscillations. A path-length oscillation produced by closed orbit distortion was experimentally identified in the NewSUBARU electron storage ring and found to be harmful to normal operations. Such oscillations are also a potential problem in other advanced light sources especially in a quasi-isochronous operation.
\end{abstract}

DOI: 10.1103/PhysRevSTAB.11.010701

PACS numbers: 29.20.-c, 29.30.Aj, 41.85.Ew, 41.85.Qg

\section{INTRODUCTION}

There are two possible external sources of longitudinal oscillation in an electron storage ring. One is a phase or voltage oscillation in the rf acceleration field and the other is a path-length oscillation produced by closed orbit distortion (COD). There have been a number of theoretical [1-4] and experimental studies [5-12] on the subject of longitudinal oscillations. Although it has long been known that a COD changes the path length and can excite longitudinal motion $[13,14]$, most published works on synchrotron radiation rings do not consider this effect. Some works using the proton storage ring studied this effect at a frequency close to the synchrotron frequency and paid no attention to a small off-resonant movement. That longitudinal movement can be more serious in the quasiisochronous operation of an electron storage ring.

The term quasi-isochronous implies an extremely small momentum compaction factor $(\alpha)$, and an extremely short equilibrium bunch length. Ideally, the equilibrium bunch length obeys a $\sqrt{\alpha}$ scaling law. The short-pulsed synchrotron radiation, generated by the short electron bunch in a ring, is used for time-resolving experiments and also as a source of strong coherent radiation in $\mathrm{THz}$ band. According to some reports of quasi-isochronous storage ring operation, however, longitudinal coherent fluctuations or oscillations can impose a practical limit on the bunch shortening. Two specific results are described below. At BESSY-II, $300 \mathrm{~Hz}$ noise was found to limit the bunch shortening because the synchrotron oscillation frequency approaches this frequency as $\alpha$ decreases [15]. At NewSUBARU, a large lower frequency energy oscillation than a few hundreds $\mathrm{Hz}$ was observed [16].

The present article shows that beam oscillations due to the rf field and magnetic deflection can be separated by the proper measurements. The sources of the coherent oscillations observed at NewSUBARU were identified and the path-length fluctuation was found to be a problem, even at off-resonance frequencies. That identification helps to eliminate the possible source of oscillation in a ring. The extrapolation for small $\alpha$ (quasi-isochronous operation) predicts a serious problem in the quasi-isochronous operation of an electron storage ring. In this article we use the word "oscillation" to express the fluctuations for a convenience of the frequency domain analysis, although offresonant movement is not always oscillatory.

\section{ANALYTICAL FORMULAS}

\section{A. Equations of longitudinal oscillation}

The longitudinal oscillation is easily calculated using the linear equations of motion [2]. The time deviation $\tau$ and the relative energy deviation $\varepsilon$ with respect to a reference particle obey the following differential equations:

$$
\begin{aligned}
& \frac{d \tau}{d t}=\alpha \varepsilon+\frac{\Delta L}{L_{0}} \\
& \frac{d \varepsilon}{d t}=-\frac{e V_{\mathrm{rf}} \omega_{\mathrm{rf}} \cos \phi_{0}}{T_{\mathrm{rev}} E_{0}}(\tau+\Delta T)-2 \alpha_{E} \varepsilon .
\end{aligned}
$$

Here $\alpha$ is the momentum compaction factor, $L_{0}$ is the circumference, and $-e$ is the electron charge. The parameters of the accelerating rf field are the voltage $V_{\mathrm{rf}}$, the angular frequency $\omega_{\mathrm{rf}}$, and the synchronous phase $\varphi_{0}$. $T_{\text {rev }}$ is the revolution period, $E_{0}$ is the energy of the reference particle, and $\alpha_{E}$ is the longitudinal damping coefficient. $\Delta T$ and $\Delta L$ are fluctuations in the rf phase and path length, respectively. A positive value of $\tau$ means a later arrival time. In the frequency domain, we should calculate complex amplitudes for one frequency component. The fluctuations are replaced by oscillating terms:

$$
\begin{aligned}
\Delta T & =\Delta_{\mathrm{rf}} e^{j \omega t}, \\
\Delta L / L_{0} & =\Delta_{L} e^{j \omega t} .
\end{aligned}
$$

Here $\omega$ is the angular frequency of the oscillation, and $\Delta_{\mathrm{rf}}$ and $\Delta_{L}$ are amplitudes. Equations (1a) and (1b) can be simplified to 


$$
\begin{aligned}
& \frac{d \tau}{d t}=\alpha \varepsilon+\Delta_{L} e^{j \omega t} \quad \text { and } \\
& \frac{d \varepsilon}{d t}=-\frac{\omega_{S}{ }^{2}}{\alpha}\left(\tau+\Delta_{\mathrm{rf}} e^{j \omega t}\right)-2 \alpha_{E} \varepsilon,
\end{aligned}
$$

where $\omega_{S}$ is the angular synchrotron oscillation frequency,

$$
\omega_{S}^{2}=\frac{e V_{\mathrm{rf}} \omega_{\mathrm{rf}} \alpha \cos \phi_{0}}{T_{\mathrm{rev}} E}
$$

The stationary solution to Eqs. (3a) and (3b) is

$$
\begin{aligned}
& \varepsilon=-\frac{\left(\omega_{S}^{2} / \alpha\right)\left(\Delta_{L}+j \omega \Delta_{\mathrm{rf}}\right)}{\omega_{S}^{2}-\omega^{2}+2 j \alpha_{E} \omega} e^{j \omega t}, \\
& \tau=\frac{\left(j \omega+2 \alpha_{E}\right) \Delta_{L}-\omega_{S}^{2} \Delta_{\mathrm{rf}}}{\omega_{S}^{2}-\omega^{2}+2 j \alpha_{E} \omega} e^{j \omega t} .
\end{aligned}
$$

Notice that the oscillation amplitude ratio $\varepsilon / \tau$ depends on the driving forces $\Delta T$ and $\Delta L$. When $\Delta_{\mathrm{rf}}$ is dominant we have

$$
\left.\frac{\varepsilon}{\tau}\right|_{\Delta L=0}=\frac{j \omega}{\alpha},
$$

and when $\Delta_{L}$ is dominant we have

$$
\left.\frac{\varepsilon}{\tau}\right|_{\Delta T=0}=-\frac{\omega_{S}{ }^{2} / \alpha}{j \omega+2 \alpha_{E}}
$$

\section{B. Path-length shift by a defection}

The shift in the average path length of a single revolution due to a slow horizontal dipole deflection can be described by a simple formula. The orbit distortion produced by a thin deflection $\theta_{S}$ is given by the well-known equation,

$$
x(s)=\frac{\sqrt{\beta_{S} \beta(s)}}{2 \sin \pi \nu} \theta_{S} \cos \left[\left|\psi(s)-\psi_{S}\right|-\pi \nu\right],
$$

where $x$ and $s$ are the horizontal and azimuthal coordinates, respectively. $\beta$ and $\psi$ are the horizontal beta function and the betatron phase, respectively, while $\beta_{S}$ and $\psi_{S}$ are their values at the deflection point. $\nu$ is the horizontal betatron tune. The shift in the path length $\Delta L_{0}$ over one revolution is thus given by

$$
\Delta L_{0}=\int_{0}^{L_{0}} \frac{x(s)}{\rho(s)} d s=\eta_{S} \theta_{S}
$$

Here $\rho$ is the radius of curvature of the reference orbit, and $\eta_{S}$ is the dispersion function at the deflection point.

When the path-length shift is much slower than the synchrotron oscillation, Eq. (5a) can be approximated as

$$
\varepsilon \approx-(1 / \alpha) \Delta_{L} e^{j \omega t} .
$$

Even in this case, the timing movement accompanies the energy movement at almost the orthogonal phase. The realized closed orbit distortion is a sum of the displacement given by Eq. (8) and the displacement due to an energy shift given by Eq. (10). That is,

$$
x(s)=\left\{\frac{\sqrt{\beta_{S} \beta(s)}}{2 \sin \pi \nu} \cos \left[\left|\psi(s)-\psi_{S}\right|-\pi \nu\right]-\frac{\eta_{S} \eta(s)}{\alpha L_{0}}\right\} \theta_{S},
$$

where $\eta$ is the dispersion function.

\section{MEASUREMENT AT NewSUBARU}

\section{A. The storage ring}

Longitudinal oscillations with harmonic frequencies equal to that of the primary power line $(60 \mathrm{~Hz}$ in western Japan) were measured in the electron storage ring at NewSUBARU [17]. The ring has six modified double bend achromat (DBA) cells, each one has one $8^{\circ}$ inverted bend (IB) between two $34^{\circ}$ normal bends. Table I presents the main parameters of NewSUBARU, and Fig. 1 shows the horizontal beta function and dispersion function in the half of the ring.

\section{B. Measurement of the energy oscillation}

The energy oscillation was identified by a measurement of a COD oscillation in half of the ring. The horizontal position signals from 9 beam position monitors (BPM4BPM12) in the eastern arc were recorded using a digital oscilloscope swept with the line trigger. The signals were averaged over 100 shots. The complex oscillation amplitude $\Delta_{X}$ was calculated at each BPM for some harmonic frequencies $\omega$. The measurement accuracy was estimated

TABLE I. Main parameters of NewSUBARU at $1 \mathrm{GeV}$.

\begin{tabular}{lc}
\hline \hline rf frequency $\left(\omega_{\text {rf }} / 2 \pi\right)$ & $499.955 \mathrm{MHz}$ \\
Synchrotron oscillation frequency $\left(\omega_{S} / 2 \pi\right)$ & $6 \mathrm{kHz}$ \\
Synchrotron oscillation damping time $\left(1 / \alpha_{\varepsilon}\right)$ & $12 \mathrm{~ms}$ \\
Circumference $\left(L_{0}\right)$ & $118.7 \mathrm{~m}$ \\
Linear momentum compaction factor $(\alpha)$ & 0.0015 \\
Natural energy spread & $0.047 \%$ \\
Horizontal betatron tune $(\nu)$ & 6.31 \\
\hline \hline
\end{tabular}

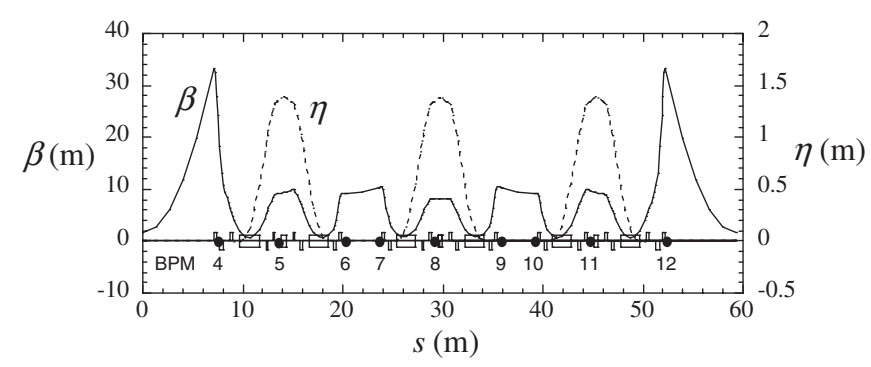

FIG. 1. The horizontal beta function $(\beta$, solid line) and dispersion function ( $\eta$, broken line) in the eastern half of the NewSUBARU ring. The boxes indicate the locations and lengths of the bending magnets and quadrupole magnets. The numbered black circles point out BPM (beam position monitors) locations. 


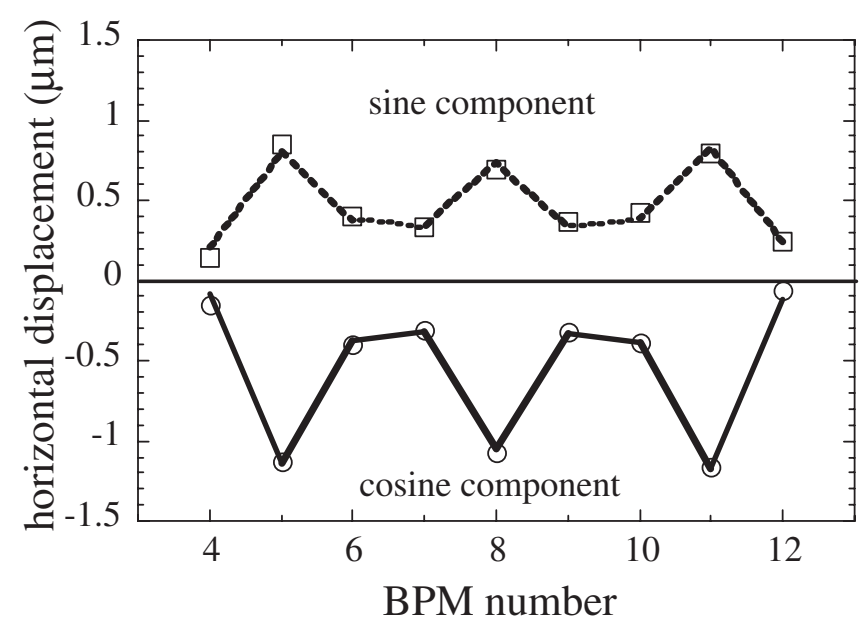

FIG. 2. The cosine and sine components of the horizontal oscillation amplitude at $180 \mathrm{~Hz}$ in the eastern arc of the ring. The cosine component is COD at the line trigger timing and the sine component is that after $1 / 720 \mathrm{sec}$. (on the diagonal phase). The clear circles and squares show measured results. The solid and broken lines are fitted functions assuming that the distortions are due to the two IB families. BPMs 4, 6, 7, 9, 10, and 12 are set in dispersion-free sections of the ring, while BPMs 5, 8, and 11 are in dispersion sections.

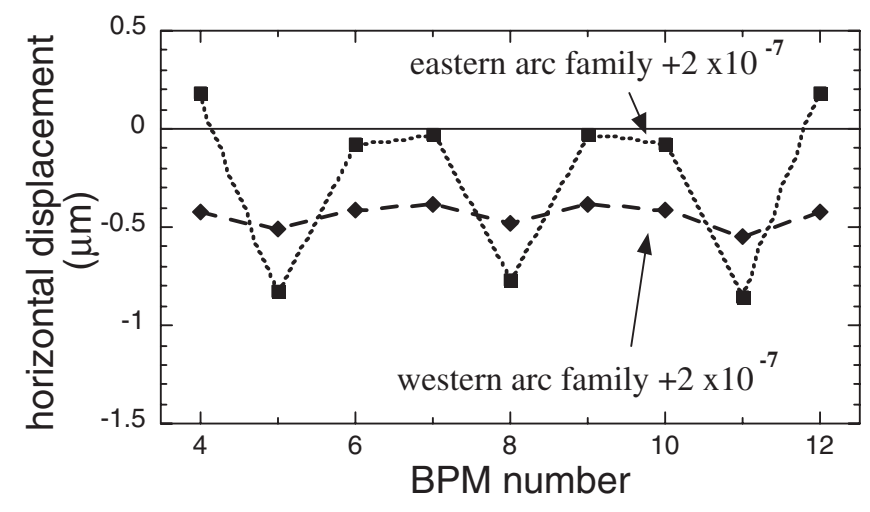

FIG. 3. Measurements of the horizontal COD under magnetic field variations due to the IB families (points). The dashed line and the dotted line show the calculated CODs produced by the IB families in the western and eastern arcs, respectively. The disagreement between the measurement and the calculation was less than $6 \%$ of the maximum. to be some tens of $\mathrm{nm}$. Such a high accuracy was obtained by picking up only a special frequency component [18]. Figure 2 shows one example of the measurements: the real and imaginary amplitude components at $180 \mathrm{~Hz}$ (the 3rd harmonic). The cosine and the sine phase components are the real and the imaginary parts of $\Delta_{X}$, respectively. The reference phase is the line trigger.

The most probable sources of the path-length oscillations in NewSUBARU are ripples of the two power supplies of the inverted bends (IBs) in the eastern arc and western arc. Figure 3 shows the horizontal CODs due to magnetic field shifts caused in these two IB families. The measured amplitude distributions throughout the arc were well fit by a combination of the theoretical COD patterns from these sources. The agreements in two orthogonal components, sine component and cosine component, assure the agreement in any phase. This strongly suggests that the energy oscillations observed at these frequencies were mainly produced by the two IB families. Figure 2 shows a clear case of this model, in which the error contributions of sources other than the two power supplies are small. Table II lists the calculated components of the energy oscillation amplitudes at 60, 120, 180, and $240 \mathrm{~Hz}$. The magnetic field fluctuations in the IB families are estimated to be roughly $10^{-7}$ of the main field. This order of the magnitude is consistent with the measured current fluctuations in the power supplies. The last row in Table II gives the expected phase oscillation amplitudes, assuming Eq. (7) holds. These values will be compared to the phase oscillation measurements described in the next subsection.

\section{Measurement of the phase oscillation}

Phase oscillation amplitudes were estimated from a fastFourier transform (FFT) power spectrum of the beam signal, which was picked up from a pickup button electrode (PUE) placed in a dispersion-free section. The signal was successive pulses, which was phase modulated by a timing modulation and amplitude modulated by a vertical orbit oscillation. The amplitudes of the modulations are obtained from the power ratio of the sideband peak to the main peak (carrier wave) at the rf frequency $\left(\omega_{\mathrm{rf}}\right)$. Some formulas for the FFT power spectrum are given in the Appendix A; Eq. (A9), for example, shows that the average

TABLE II. Field oscillation and longitudinal oscillation amplitudes estimated from the orbit oscillation.

\begin{tabular}{|c|c|c|c|c|c|}
\hline \multicolumn{2}{|c|}{ Harmonic frequency; $\omega / 2 \pi(\mathrm{Hz})$} & \multirow{2}{*}{$\frac{60}{3.1}$} & \multirow{2}{*}{$\frac{120}{6}$} & \multirow{2}{*}{$\frac{180}{3}$} & \multirow{2}{*}{$\frac{240}{0.4}$} \\
\hline IB field ripple $\left(10^{-7}\right)$ & Eastern arc family & & & & \\
\hline & Western arc family & 1.3 & 4 & 1.9 & 0.5 \\
\hline \multicolumn{2}{|c|}{ Energy oscillation amplitude; $|\varepsilon|\left(10^{-6}\right)$} & 1.1 & 1.2 & 1.3 & 0.3 \\
\hline \multicolumn{2}{|c|}{ Path-length oscillation amplitude; $\left|\Delta_{L}\right|\left(10^{-9}\right)$} & 1.7 & 1.8 & 2.0 & 0.4 \\
\hline \multicolumn{2}{|c|}{ Expected phase oscillation amplitude; $|\tau|$ (fs) } & 0.7 & 1.4 & 2.4 & 0.6 \\
\hline
\end{tabular}


TABLE III. Ratio of sideband peak height to main peak height (carrier wave) in the FFT power spectrum.

\begin{tabular}{lcccc}
\hline \hline \multicolumn{1}{c}{ Harmonic frequency $(\mathrm{Hz})$} & 60 & 120 & 180 & 240 \\
\hline Phase oscillation amplitude; $|\tau|(\mathrm{fs})$ & 31 & 33 & 6 & 24 \\
Expected energy oscillation amplitude; $|\varepsilon|\left(10^{-6}\right)$ & 0.008 & 0.018 & 0.005 & 0.026 \\
\hline \hline
\end{tabular}

power ratio of the two sideband peaks (at $\omega_{\text {rf }}+\omega$ and $\left.\omega_{\mathrm{rf}}-\omega\right)$ is equal to the total power of the two modulations.

Table III shows the calculated timing (phase) modulation amplitudes of the $60,120,180$, and $240 \mathrm{~Hz}$ components. The details of this calculations, including estimation of the vertical orbit oscillation and subtraction of its contribution, are explained in Appendix B. These phase modulations were larger than those expected from the pathlength oscillation measurements listed in Table II. This means that the main source of beam phase oscillation is the ripples in the rf accelerating field. Table III also gives an estimation of the energy oscillation amplitudes produced by these ripples, assuming Eq. (6) holds. These results are much smaller than the measurements listed in Table II. The contribution of the ripples to the energy oscillation is therefore very small, and it is not necessary to change the main results of the previous subsection. The main source of the energy fluctuation is the field fluctuations of the inverted bends.

\section{DISCUSSION}

\section{A. The effect of path-length fluctuations at NewSUBARU}

In the normal operations at NewSUBARU, it has become clear that the main source of low-frequency energy fluctuations is the path-length fluctuation and that of the beam phase fluctuation is the rf system. At present, the energy fluctuation is harmful because it introduces an uncertainty into the COD measurement.

The slow COD measurement system of NewSUBARU has one analogue to digital converter (ADC) for each bending arc. The demodulated signals from the BPM electrodes are selected one by one by a switching unit and are detected by the ADC. Consequently, fluctuation of the horizontal beam position produces an uncertainty in both the horizontal and vertical directions. The low-frequency energy oscillation is the main source of uncertainty in the vertical COD measurement at points, where the dispersion is large and the vertical beta function is small. The synchrotron oscillation amplitude is larger, but not harmful because its frequency is high enough to be blocked by a low pass filter.

\section{B. Effect in quasi-isochronous operation}

Figure 4 shows the expected $\alpha$ dependence of the energy and phase oscillation amplitudes at NewSUBARU. The oscillation amplitudes in energy and time produced by the path-length oscillation are expected to be larger than the natural spread under quasi-isochronous conditions. The phase fluctuation amplitude grows larger for smaller momentum compaction factor $\alpha$. This explains the strange $\alpha$ dependence of the bunch length previously measured at NewSUBARU [16]. We had expected a $\sqrt{\alpha}$ scaling law for the bunch length, but for $\alpha<5 \times 10^{-5}$ the bunch length was even longer for smaller $\alpha$.
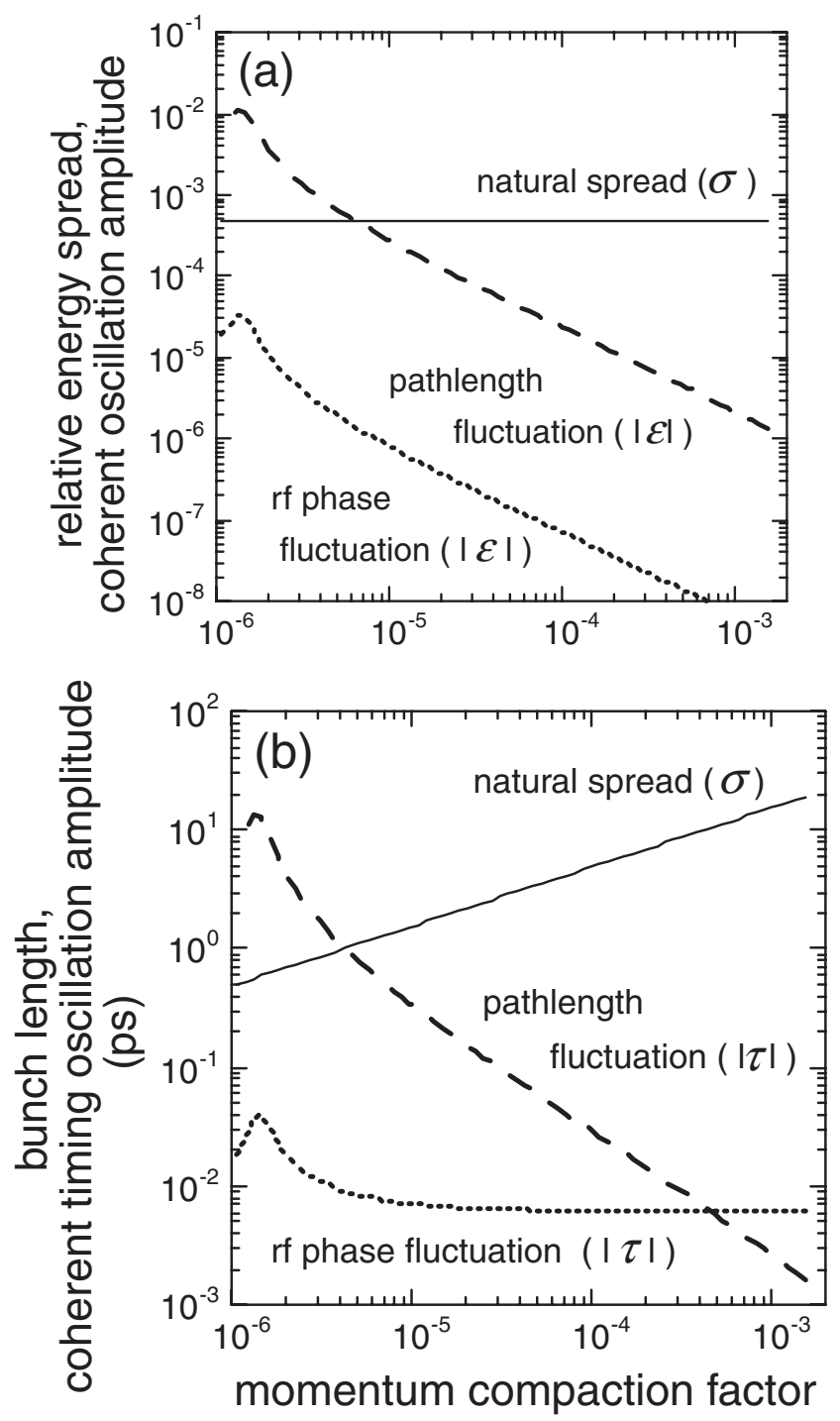

FIG. 4. Estimation of the energy oscillation (a) and phase oscillation (b) amplitudes at $180 \mathrm{~Hz}$, as a function of the momentum compaction factor. 
In another quasi-isochronous ring, such as BESSTY-II, ripples in the bending magnet power supply are less harmful for two reasons. (i) A normal DBA ring has only one bending magnet family, so a field change in the magnets produces an energy shift but not an orbital displacement; (ii) The path-length shift of NewSUBARU has little $\alpha$ dependence, but that of BESSY-II is proportional to $\alpha$.

For dipole field fluctuations arising from something other than a bending magnet, the $\alpha$ dependence of the amplitude is related to the $\alpha$ dependence of the dispersion. We consider the following three cases. (a) The dispersion $\eta$ is roughly constant with respect to $\alpha$. This is the case for the IB family of NewSUBARU, and could represent field fluctuations at a dispersion section of the normal DBA lattice. (b) $\eta$ is proportional to $\alpha$. This is the case for the bending magnets of the normal DBA lattice, assuming that they have more than one power supply. (c) $\eta$ is larger for smaller $\alpha$. This is the case for a straight section of the normal DBA lattice, where the achromatic condition is broken and $\alpha$ decreases. This kind of dependence is written as $\eta \propto \alpha-\alpha_{0}$, where $\alpha_{0}$ is the momentum compaction factor under achromatic conditions. In this case nonzero dispersion in an rf cavity produces a COD that depends on the rf acceleration $[19,20]$. However, in this article we ignore this effect. Figure 5 shows the $\alpha$ dependence of the phase oscillation amplitude for the three cases. The path-length oscillation is always more harmful at lower $\alpha$.

A coherent phase fluctuation does not always enlarge the instantaneous bunch length, which determines the coherent radiation spectrum, but it does reduce the accuracy of timeresolving experiments. In some cases, where the ring has a large higher order momentum compaction factor, the large energy fluctuations can effectively enlarge the momentum

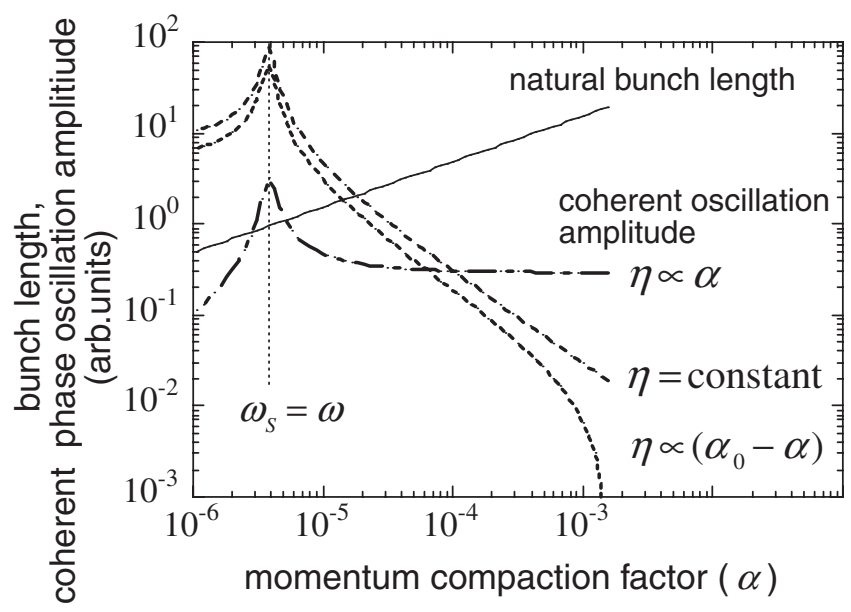

FIG. 5. Dependence of coherent phase oscillation amplitude on momentum compaction factor $(\alpha)$. The natural dependence of the bunch length is shown as a solid line. The other lines present the coherent oscillation amplitudes due to a dipole field error at three locations. The amplitudes are set to show the $\alpha$ dependence clearly. compaction factor [21] or induce parametric resonances $[3,4]$.

\section{SUMMARY}

Low-frequency longitudinal coherent oscillations in NewSUBARU were identified by this analysis. These oscillations are harmful to daily operations and are especially serious when the momentum compaction factor is small. This effect explains the strange behavior of the bunch length, which was found to increase for smaller momentum compaction factors. The method explained in the article is useful for distinguishing rf noise from magnetic noise.

\section{ACKNOWLEDGMENTS}

The authors thank Dr. T. Ohshima of SPring- 8 for his help with the measurements and many useful suggestions.

\section{APPENDIX A: SIDEBAND PEAK POWER IN THE FFT SPECTRUM}

The beam signal picked up by a monitor electrode consists of successive pulses. Its amplitude is modulated by the orbit displacement and timing is modulated by the phase oscillation. Assuming the uniform filling of rf buckets, the beam signal can thus be approximated as

$$
V(t)=\left(V_{0} T_{\mathrm{rf}}\right)(1+\kappa y) \delta\left[\omega_{\mathrm{rf}}(t+\tau)-2 n \pi\right]
$$

Here $\delta[t]$ is the Dirac delta function, based on the assumption of a negligibly short electron bunch. $V$ is the signal voltage of a pulse and $T_{\text {rf }}$ is the rf period, so $V_{0} T_{\text {rf }}$ is the integrated voltage of a pulse with no modulation. $\kappa$ is a coefficient describing the signal response for a given orbital displacement $y . \omega_{\text {rf }}$ is the angular frequency of the $\mathrm{rf}$ acceleration field. $\tau$ is the time displacement relative to a reference value, and $n$ is the pulse number approximated by $n \approx t / T_{\mathrm{rf}}$. The coefficient $\kappa$ is determined from the geometrical structure of the monitor.

The modulation amplitude determines the height of the sideband in the FFT spectrum. We assume that the angular rf frequency (carrier wave) is an integer times the modulation frequency $\omega, \omega_{\mathrm{rf}}=N \omega$. The Fourier amplitudes of the sidebands, with angular frequencies $\omega_{\mathrm{rf}}+\omega$ and $\omega_{\mathrm{rf}}-$ $\omega$, are given by the following equation:

$$
V_{\omega_{\mathrm{rf}} \pm \omega}{ }^{( \pm)}=(1 / T) \int_{0}^{T} V(t) \exp \left[( \pm) j\left(\omega_{\mathrm{rf}} \pm \omega\right) t\right] d t .
$$

$T$ is the modulation period, $T=N T_{\mathrm{rf}}$. Substituting Eq. (A1) into (A2) gives 


$$
\begin{aligned}
V_{\omega_{\mathrm{rf}} \pm \omega}( \pm)= & \frac{V_{0}}{N} \sum_{n=1}^{N}(1+\kappa y) \\
& \times \exp \left[( \pm) j\left(\omega_{\mathrm{rf}} \pm \omega\right)\left(2 \pi n / \omega_{\mathrm{rf}}-\tau\right)\right] \\
= & \frac{V_{0}}{N} \sum_{n=1}^{N}(1+\kappa y) \\
& \times \exp \left[( \pm) j\left( \pm 2 \pi n / N-\left(\omega_{\mathrm{rf}} \pm \omega\right) \tau\right)\right] \\
\approx & \frac{V_{0}}{N} \sum_{n=1}^{N}\left[\kappa y-( \pm) j\left(\omega_{\mathrm{rf}} \pm \omega\right) \tau\right] e^{( \pm) \pm j \omega t} .
\end{aligned}
$$

We define the modulation amplitudes $\Delta_{y}$ and $\Delta_{\tau}$ by

$$
\begin{aligned}
& y=\Delta_{y} e^{j \omega t}, \\
& \tau=\Delta_{\tau} e^{j \omega t} .
\end{aligned}
$$

Substituting them into Eq. (A3) gives the four components

$$
\begin{aligned}
& V_{\omega_{\mathrm{rf}}+\omega}{ }^{(+)} \approx 0, \\
& V_{\omega_{\mathrm{rf}}+\omega}{ }^{(-)} \approx V_{0}\left[\kappa \Delta_{y}+j\left(\omega_{\mathrm{rf}}+\omega\right) \Delta_{\tau}\right], \\
& V_{\omega_{\mathrm{rf}}-\omega}{ }^{(+)} \approx V_{0}\left[\kappa \Delta_{y}-j\left(\omega_{\mathrm{rf}}-\omega\right) \Delta_{\tau}\right], \\
& V_{\omega_{\mathrm{rf}}-\omega}{ }^{(-)} \approx 0 .
\end{aligned}
$$

The height of the sidebands relative to the main peak is given by

$$
\left|\frac{V_{\omega_{\mathrm{rf}} \pm \omega}}{V_{0}}\right| \approx\left|\kappa \Delta_{y} \pm j\left(\omega_{\mathrm{rf}} \pm \omega\right) \Delta_{\tau}\right|
$$

Assuming $V_{0} \approx V_{\omega_{\mathrm{rf}}}$ and $\omega \ll \omega_{\mathrm{rf}}$, the above equation is approximated by

$$
\left|\frac{V_{\omega_{\mathrm{rf}} \pm \omega}}{V_{\omega_{\mathrm{rf}}}}\right| \approx\left|\kappa \Delta_{y} \pm j \omega_{\mathrm{rf}} \Delta_{\tau}\right| .
$$

Using the amplitude ratios $\left|V_{\omega_{\mathrm{rf}}+\omega} / V_{0}\right|$ and $\left|V_{\omega_{\mathrm{rf}}-\omega} / V_{0}\right|$, we obtain possible maximum and minimum values for each modulation amplitude:

$$
\begin{gathered}
\left|\frac{\left|V_{\omega_{\mathrm{rf}}+\omega} / V_{0}\right|-\left|V_{\omega_{\mathrm{rf}}-\omega} / V_{0}\right|}{2}\right| \leq\left|\kappa \Delta_{y}\right|, \\
\left|\omega_{\mathrm{rf}} \Delta_{\tau}\right| \leq \frac{\left|V_{\omega_{\mathrm{rf}}+\omega} / V_{0}\right|+\left|V_{\omega_{\mathrm{rf}}-\omega} / V_{0}\right|}{2} .
\end{gathered}
$$

The average power ratio of the two sidebands is equal to the sum of the phase modulation and the amplitude modulation powers:

$$
\frac{\left|V_{\omega_{\mathrm{rf}}+\omega} / V_{0}\right|^{2}+\left|V_{\omega_{\mathrm{rf}}-\omega} / V_{0}\right|^{2}}{2}=\left|\kappa \Delta_{y}\right|^{2}+\left|\omega_{\mathrm{rf}} \Delta_{\tau}\right|^{2} \text {. }
$$

We first measured $\left|V_{\omega_{\mathrm{rf}}+\omega} / V_{0}\right|,\left|V_{\omega_{\mathrm{rf}}-\omega} / V_{0}\right|$, and $\left|\kappa \Delta_{y}\right|$, then obtained $\left|\omega_{\text {rf }} \Delta_{\tau}\right|$ using Eq. (A9). In this article, as our measurement of $\left|\kappa \Delta_{y}\right|$ has a large uncertainty, Eq. (A8) is used to check that the values were consistent (Appendix B).
Equation (A7) can also be used to identify the source of the fluctuation in a special case, when the monitor electrode is placed in a dispersion section and is sensitive to the horizontal displacement, and when the vertical COD fluctuations are negligible. The displacement $y$ in Eq. (A1) can then be replaced by a horizontal displacement, which is proportional to the energy displacement $(\varepsilon)$. In that case Eq. (A7) becomes

$$
\left|\frac{V_{\omega_{\mathrm{rf}} \pm \omega}}{V_{\omega_{\mathrm{rf}}}}\right| \approx\left|\kappa k \Delta_{\varepsilon} \pm j \omega_{\mathrm{rf}} \Delta_{\tau}\right|
$$

where $k$ is the coefficient relating the horizontal displacement to the energy displacement and $\Delta_{\varepsilon}$ is the energy oscillation amplitude. Substituting Eqs. (5a) and (5b) into Eq. (A10) makes

$$
\begin{aligned}
& \left|\frac{V_{\omega_{\mathrm{rf}} \pm \omega}}{V_{\omega_{\mathrm{rf}}}}\right| \\
& \approx\left|\frac{\left(\kappa k \omega_{S}^{2} / \alpha\right)\left(\Delta_{L}+j \omega \Delta_{\mathrm{rf}}\right) \pm \omega_{\mathrm{rf}}\left(\omega \Delta_{L}+j \omega_{S}^{2} \Delta_{\mathrm{rf}}\right)}{\omega_{S}^{2}-\omega^{2}+2 j \alpha_{E} \omega}\right| .
\end{aligned}
$$

In this step we assumed $\alpha_{E} \ll \omega$. When $\Delta T$ is dominant $\left(\Delta_{L}=0\right)$, the imbalance of the two sidebands should be

$$
\left|\frac{V_{\omega_{\mathrm{rf}} \pm \omega}}{V_{\omega_{\mathrm{rf}}-\omega}}\right| \approx\left|\frac{\kappa k \omega+\alpha \omega_{\mathrm{rf}}}{\kappa k \omega-\alpha \omega_{\mathrm{rf}}}\right| .
$$

Equation (A12) does not have an explicit $V_{\mathrm{rf}}$ dependence. On the other hand, when $\Delta L$ is dominant the imbalance is given by

$$
\left|\frac{V_{\omega_{\mathrm{rf}}+\omega}}{V_{\omega_{\mathrm{rf}}-\omega}}\right| \approx\left|\frac{\kappa k \omega_{S}^{2}+\alpha \omega \omega_{\mathrm{rf}}}{\kappa k \omega_{S}^{2}-\alpha \omega \omega_{\mathrm{rf}}}\right|
$$

This is different from (A12), and has a $V_{\mathrm{rf}}$ dependence through $\omega_{S}$. The coefficient $k$ is determined from Eq. (11), which depends on the locations of the fluctuation source and the monitor electrode. Equations (A12) and (A13) show that the imbalance of the sidebands contains important information, and these relations can be used to identify the source of the fluctuation.

\section{APPENDIX B: ESTIMATION OF VERTICAL BEAM ORBIT OSCILLATION AT PICKUP ELECTRODE}

This Appendix explains how the vertical orbit oscillation at the PUE was estimated and subtracted from the results of FFT.

The vertical oscillation was estimated from the vertical COD at the modulation frequency; from this, we obtain the phase modulation amplitude. Figure 6 shows an example of the vertical COD components at $60 \mathrm{~Hz}$ fit with sinusoidal functions of the betatron phase. The vertical position signal was 2.5 times noisy than that of the horizontal one because of the geometrical structure of the BPM. However, the deviations from the fit, including the systematic deviation, 


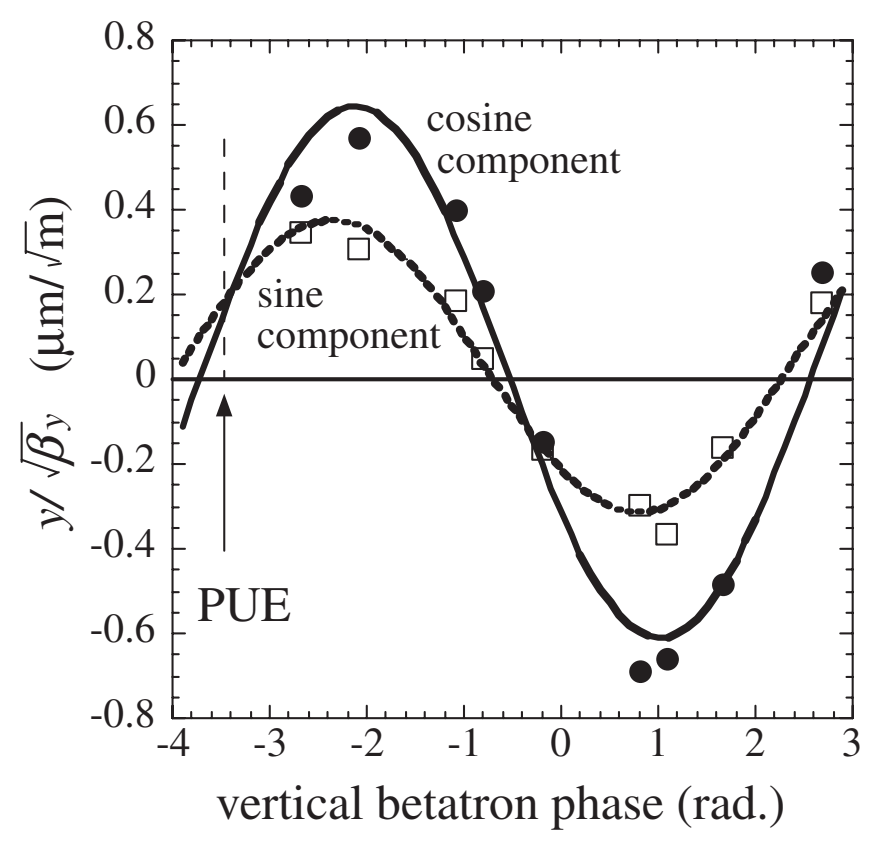

FIG. 6. The cosine and sine components of the vertical oscillation amplitude at $60 \mathrm{~Hz}$, measured in the eastern arc. The PUE is located between the two arcs, where the arrow indicates. The sinusoidal lines are the best-fit functions for betatron motion, assuming that there are no vertical dipole errors in this section.

were smaller than $0.15 \mu \mathrm{m} / \mathrm{m}^{-1 / 2}$. The oscillation amplitude at the PUE was calculated by extrapolating the function.

This estimation is not very accurate, because it assumes that there is no source of error in that area. Then a consistency of the data was checked against the upper and lower bounds obtained from the FFT spectrum, using Eq. (A8). Table IV shows the values for modulations with $60,120,180$, and $240 \mathrm{~Hz}$. In all cases the estimated vertical oscillation amplitudes $\left|\kappa \Delta_{y}\right|$ lay between the maximum and minimum values given by Eq. (A8). At $60 \mathrm{~Hz}$ the power imbalance of the sidebands was considerable (3.7 dB) and $\left|\kappa \Delta_{y}\right|$ and $\left|\omega_{\text {rf }} \Delta_{\tau}\right|$ were of the same order. At 180 and $240 \mathrm{~Hz}$, on the other hand, the imbalances were only 0.9 and $1.2 \mathrm{~dB}$. Thus, it was reasonable that $\left|\kappa \Delta_{y}\right|$ was much smaller than $\left|\omega_{\mathrm{rf}} \Delta_{\tau}\right|$.

The timing oscillation amplitudes, $|\tau| \equiv\left|\Delta_{\tau}\right|$, listed in Table III, were calculated from values listed in Table IV using Eq. (A9).

[1] S. Daté, K. Soutome, and A. Ando, Nucl. Instrum. Methods Phys. Res., Sect. A 355, 199 (1995).

[2] S. Y. Lee, Accelerator Physics (World Scientific, Singapore, 1999), Chap. 3.III, p. 254.

[3] A. Riabko, M. Bai, B. Brabson, C. M. Chu, X. Kang, D. Jeon, S. Y. Lee, and X. Zhao, Phys. Rev. E 54, 815 (1996).

[4] M. Bai, D. Jeon, S. Y. Lee, K. Y. Ng, A. Riabko, and X. Zhao, Phys. Rev. E 55, 3493 (1997).

[5] M. Syphers, M. Ball, B. Brabson, J. Budnick, D. D. Caussyn, A. W. Chao, J. Collins, V. Derenchuk, S. Dutt, G. East, M. Ellison, T. Ellison, D. Friesel, W. Gabella, B. Hamilton, H. Huang, W. P. Jones, S. Y. Lee, D. Li, M. G. Minty, S. Nagaitsev, K. Y. Ng, X. Pei, G. Rondeau, T. Sloan, L. Teng, S. Tepikian, Y. Wang, Y. T. Yan, and P. L. Zhang, Phys. Rev. Lett. 71, 719 (1993).

[6] H. Huang, M. Ball, B. Brabson, J. Budnick, D. D. Caussyn, A. W. Chao, J. Collins, V. Derenchuk, S. Dutt, G. East, M. Ellison, D. Friesel, B. Hamilton, W. P. Jones, S. Y. Lee, D. Li, M. G. Minty, S. Nagaitsev, K. Y. Ng, X. Pei, A. Riabko, T. Sloan, M. Syphers, L. Teng, Y. Wang, Y. T. Yan, and P. L. Zhang, Phys. Rev. E 48, 4678 (1993).

[7] Y. Wang, M. Ball, B. Brabson, J. Budnick, D. D. Caussyn, A. W. Chao, J. Collins, V. Derenchuk, S. Dutt, G. East, M. Ellison, D. Friesel, B. Hamilton, H. Huang, W. P. Jones, S. Y. Lee, D. Li, J. Y. Liu, M. G. Minty, K. Y. Ng, X. Pei, A. Riabko, T. Sloan, M. Syphers, L. Teng, Y. T. Yan, and P. L. Zhang, Phys. Rev. E 49, 1610 (1994).

[8] M. Hara, T. Nakamura, and T. Ohshima, Part. Accel. 59, 143 (1998).

[9] K.W. Ormond and T.J. Rogers, Proceedings of the Particle Accelerator Conference, Vancouver, Canada, 1997, pp. 1822-1824.

[10] S. Sakanaka, M. Izawa, T. Obina, and T. Takahashi, Proceedings of the Asian Particle Accelerator Conference, Beijing, China, 2001, pp. 454-456.

TABLE IV. The measured ratio of sideband peak height to main (carrier wave) peak height in the FFT power spectrum. The estimated vertical oscillation amplitude $\left|\kappa \Delta_{y}\right|$ was compared to the upper and lower limits given by the FFT power spectrum. The errors for $\left|\Delta_{y}\right|$ and $\left|\kappa \Delta_{y}\right|$ were the fitting errors.

\begin{tabular}{llrrrr}
\hline \hline \multicolumn{2}{c}{ Harmonic frequency $(\mathrm{Hz})$} & 60 & 120 & 180 & 240 \\
\hline Sideband peak height $\left(10^{-6}\right)$ & $\left|V_{\omega_{\mathrm{rf}}-\omega} / V_{\omega_{\mathrm{rf}}}\right|$ & 128 & 120 & 19 & 82 \\
& $\left|V_{\omega_{\mathrm{rf}}}\right| V_{\omega_{\mathrm{rf}}} \mid$ & 84 & 96 & 21 & 71 \\
Possible maximum $\left(10^{-6}\right)$ & & 106 & 108 & 20 & 76 \\
Possible minimum $\left(10^{-6}\right)$ & & 22 & 12 & 1 & 5 \\
Vertical oscillation amplitude & $\left|\Delta_{y}\right|\left(10^{-8} \mathrm{~m}\right)$ & $46 \pm 23$ & $31 \pm 8$ & $1 \pm 9$ & $8 \pm 5$ \\
& $\left|\kappa \Delta_{y}\right|\left(10^{-6}\right)$ & $48 \pm 25$ & $33 \pm 9$ & $1 \pm 10$ & $8 \pm 6$ \\
\hline \hline
\end{tabular}


[11] Y. Shoji, A. Ando, H. Ego, Y. Kawashima, T. Ohshima, and T. Takashima, Proceedings of the Asian Particle Accelerator Conference, Geongju, Korea, 2004, pp. $368-370$.

[12] T. Ohshima and N. Kumagai, Proceedings of the Particle Accelerator Conference, Chicago, USA, 2001, pp. 19751977.

[13] A. Piwinski and A. Wrulich, DESY 76/07, 1976; A. Piwinski, Handbook of Accelerator Physics and Engineering, edited by $\mathrm{A}$. $\mathrm{Wu}$ Chao and $\mathrm{M}$. Tigner (World Scientific, Singapore, 1999), Chap. 2, p. 72.

[14] T. Suzuki, Phys. Rev. Lett. 96, 214801 (2006).

[15] J. Feikes, K. Holldack, P. Kuske, and G. Wüestefeld, Proceedings of the European Particle Accelerator Conference, Luzern, Switzerland, 2004, pp. 1954-1956.
[16] Y. Shoji, S. Hisao, and T. Matsubara, Proceedings of the European Particle Accelerator Conference, Lucerne, Switzerland, 2004, pp. 2356-2357.

[17] A. Ando, A. Amano, S. Hashimoto, H. Kinoshita, S. Miyamoto, T. Mochizuki, M. Niibe, Y. Shoji, M. Terasawa, T. Watanabe, and N. Kumagai, J. Synchrotron Radiat. 5, 342 (1998).

[18] T. Nakatani, A. Agui, H. Aoyagi, T. Matsushita, M. Takao, M. Takeuchi, A. Yoshigoe, and H. Tanaka, Rev. Sci. Instrum. 76, 055105 (2005).

[19] W. Guo, K. Harkay, and M. Borland, Phys. Rev. E 72, 056501 (2005).

[20] X. Huang, Phys. Rev. ST Accel. Beams 10, 014002 (2007).

[21] Y. Shoji, Phys. Rev. ST Accel. Beams 8, 094001 (2005). 